\title{
Is the liberal use of preoperative 3-dimensional imaging and presternotomy femoral cutdown beneficial in reoperative adult congenital heart surgery?
}

\author{
Brian E. Kogon, MD, ${ }^{a}$ William Daniel, MD, ${ }^{a}$ Katherine Fay, MD, ${ }^{a}$ and Wendy Book, MD $^{b}$
}

Objective: Patients with congenital heart disease frequently survive into adulthood, and many of them will require repeat surgery. Often, the unique anatomy can make reoperative sternotomy and the conduct of cardiopulmonary bypass challenging. We evaluated the utility of preoperative 3-dimensional imaging and presternotomy femoral cutdown in reoperative adult congenital heart disease surgery.

Methods: We retrospectively studied 205 adult patients, who had undergone reoperative cardiac surgery for congenital heart disease from 2006 to 2011. Using the operative history and 3-dimensional preoperative imaging findings, an algorithm was created to determine whether femoral cutdown or cannulation should be performed before sternal reentry. Analyses were performed to determine the benefits of this strategy. In addition, analyses were performed to identify adverse outcomes related to this strategy.

Results: Presternotomy femoral intervention was performed in 112 of 205 patients $(55 \%$ ) -femoral cutdown alone in $69(34 \%)$ and femoral cutdown, cannulation, and institution of cardiopulmonary bypass in $43(21 \%)$. Of the 19 patients $(9 \%)$ with a cardiac injury, femoral cutdown had already been performed in 17, of whom 10 had also undergone cannulation. Only 2 patients required urgent femoral cutdown or cannulation. A strong correlation was found between the site of injury predicted by the preoperative algorithm and the actual site of cardiac injury $(88 \%)$. In both univariate and multivariate models, the risk factors for cardiac injury included a history of cardiac injury during sternal reentry $(18 \%$ vs $1 \%, P=.0001)$, proximity of the right ventricular outflow tract to the posterior chest wall ( $35 \%$ vs $14 \%, P=.04)$, and increased reoperative sternotomy incidence $(P=.01)$. In 31 patients, despite safe reentry, the femoral vessels were used as a preferential site of venous $(n=6)$, arterial $(n=9)$, or venous and arterial cannulation $(n=16)$ because of anatomic constraints within the chest cavity. Three patients experienced groin complications (pseudoaneurysm, abscess, ischemia) requiring surgery.

Conclusions: Cardiac injury during reoperative surgery in adults with congenital heart disease is not uncommon. The preoperative history and imaging findings could be predictive of certain cardiac injury patterns. Using the preoperative history and 3-dimensional imaging findings, a more selective algorithm for presternotomy femoral intervention might be warranted. (J Thorac Cardiovasc Surg 2014;147:1799-804)

Many patients with congenital heart disease will require repeat surgery after surviving into adulthood. These operations can be extremely difficult because of these patients' unique anatomy and previous scarring. We evaluated the utility of preoperative 3-dimensional imaging and presternotomy femoral cutdown in reoperative adult congenital heart disease surgery.

\section{METHODS}

We performed a retrospective cohort study of 205 consecutive adult patients with a history of congenital heart disease, who had undergone reoperative cardiac surgery at a single, large, academic center from

\footnotetext{
From the Departments of Cardiothoracic Surgery ${ }^{\mathrm{a}}$ and Cardiology, ${ }^{\mathrm{b}}$ Emory University School of Medicine, Atlanta, Ga.

Disclosures: Authors have nothing to disclose with regard to commercial support. Received for publication Dec 2, 2012; revisions received July 4, 2013; accepted for publication July 26, 2013; available ahead of print Sept 25, 2013.

Address for reprints: Brian E. Kogon, MD, Department of Cardiothoracic Surgery,

Emory University School of Medicine, Children's Healthcare of Atlanta at

Egleston, Atlanta, GA 30306 (E-mail: Bkogon@emory.edu).

$0022-5223 / \$ 36.00$

Copyright $\odot 2014$ by The American Association for Thoracic Surgery

http://dx.doi.org/10.1016/j.jtcvs.2013.07.074
}

2006 to 2011. All operations were performed by a single surgeon. The institutional review board approved the present retrospective study, and individual patient consent was waived.

The operative history and preoperative imaging findings (computed tomography $[\mathrm{CT}]$ or magnetic resonance imaging) were obtained for al patients. Using this information, an algorithm (Figure 1) was created to determine whether (1) no femoral intervention, (2) femoral cutdown alone, or (3) femoral cutdown, cannulation, and institution of cardiopulmonary bypass should be performed before sternal reentry. Femoral cutdown, cannulation, and institution of cardiopulmonary bypass were performed if a difficult reentry or cardiac injury during a previous sternotomy $(n=4)$ had been reported. Otherwise, femoral intervention was determined by the anatomy of the immediate retrosternal space. If the aorta or righ ventricular outflow tract was positioned in the immediate retrosterna space, femoral cutdown, cannulation, and institution of cardiopulmonary bypass were performed $(n=39)$. If the right atrium or right ventricle was positioned in the immediate retrosternal space, femoral cutdown alone was performed $(n=69)$. If a clear space was present between the sternum and the heart, no presternotomy intervention was performed $(\mathrm{n}=93)$.

\section{Femoral Cutdown and Cannulation Technique}

A transverse incision was made in the groin approximately $1 \mathrm{~cm}$ above the groin crease. The common femoral artery and vein were isolated. Heparin was administered (5000 IU), and an 8-mm Dacron tube graft 


\section{Abbreviation and Acronym \\ $\mathrm{CT}=$ computed tomography}

was sewn end-to-side into the artery. The tube graft was then cannulated, typically with a $20 \mathrm{~F}$ or $22 \mathrm{~F}$ reoperative aortic cannula, allowing bidirectional perfusion throughout the procedure. At the end of the case, the tube graft was clamped and oversewn, leaving a small cuff attached to the femoral artery.

\section{Reoperative Sternotomy Technique}

Repeat sternotomy was performed in a standard fashion by the attending surgeon. The soft tissues were opened, and previously placed sternal wires were removed completely. The xyphoid process was split and cleared on its undersurface. Bilateral rake retractors were then used to elevate each hemisternum anteriorly. Using electrocautery, the underlying structures were dissected from the posterior chest wall as far as could be reached with direct visualization. The oscillating saw was then used to divide the sternum, but only as far as the previous dissection allowed. This process of dissecting and sawing was repeated, working in a cephalad direction until the sternum was opened completely. Once the sternum was opened, the sternal retractor could be safely positioned, and internal dissection could proceed.

\section{Outcomes}

Operative mortality, the presence of a major adverse event, and cardiac injury were chosen as outcomes. Operative mortality was defined as death occurring within the surgical hospital stay or within 30 days after surgery. Our major adverse event composite outcome variable was positive if the patient experienced any of the following major complications: stroke, renal failure, prolonged ventilation, deep sternal infection, reoperation, and operative mortality. Cardiac injury was defined by entry into a cardiac chamber or mediastinal great vessel (vein or artery).

\section{Statistical Analysis}

Summary statistics were performed. Univariate and multivariate risk factor models for cardiac injury were created using the preoperative history and imaging findings. Outcome comparisons were then made between those patients who had undergone presternotomy femoral intervention and those who had not. A Mann-Whitney $U$ test was used for continuous variables and a chi-square test for categorical variables. Significant associations were based on $P<.05$.

\section{RESULTS}

\section{Summary Statistics}

For our cohort of 205 patients, the median age was 27 years (range, 18-72), and their median weight was $70 \mathrm{~kg}$ (range, 36-143). Other demographic, preoperative, and operative characteristics are summarized in Table 1.

\section{Cardiac Injury}

Cardiac injury occurred in 19 patients (9\%; Figure 2). Injury to the right ventricular outflow tract was most common $(n=10)$, followed by the innominate vein $(n=3)$, aorta $(\mathrm{n}=2)$, right ventricle $(\mathrm{n}=2)$, and right atrium $(\mathrm{n}=2)$.

\section{Relationship Between Femoral Intervention and Cardiac Injury}

The outcomes stratified by intervention group are shown in (Figure 3).
Of the 205 patients, $93(45 \%)$ underwent no femoral intervention before sternal reentry. Of these 93 patients, $91(98 \%)$ underwent safe sternal reentry, and 2 sustained a cardiac injury (innominate vein in both).

Of the 205 patients, $69(34 \%)$ underwent femoral cutdown alone and $43(21 \%)$ underwent or femoral cutdown, cannulation, and institution of cardiopulmonary bypass before reoperative sternotomy. Of the 19 patients who sustained a cardiac injury, femoral cutdown had already been performed in 17 patients, of whom 10 had also undergone cannulation. Only 2 patients (previously mentioned) required urgent femoral cutdown and/or cannulation.

\section{Correlation and Risk Factors}

A strong correlation was found between the site of injury predicted by the preoperative algorithm and the actual site of cardiac injury (Table 2). After subtracting the 3 innominate vein injuries (a structure not included in the algorithm), the site of injury predicted by history and imaging correlated with the actual site of injury in 14 of 16 patients $(88 \%)$.

In a univariate model analyzing the parameters from the algorithm, the significant risk factors for cardiac injury included a history of cardiac injury during sternal reentry (75\% vs $7 \%, P=.0001)$, the proximity of the right ventricular outflow tract to the posterior chest wall $(19 \%$ vs $6 \%, P=.04)$, and an increasing number of previous sternotomies $(P=.01$; Table 3$)$. All 3 risk factors maintained significance in the multivariate model.

\section{Femoral Cannulation}

Ultimately, the femoral vessels were cannulated for cardiopulmonary bypass in 84 of the 205 patients. Venous and arterial cannulation was used in 19 patients for cardiopulmonary support because of cardiac injury and in 33 patients to facilitate a successful safe reentry. In 31 patients, despite a safe reentry, the femoral vessels were used as a preferential site of venous $(\mathrm{n}=6)$, arterial $(n=9)$, or venous and arterial cannulation $(n=16)$ because of anatomic constraints within the chest cavity.

\section{Complications}

Three patients developed groin complications (pseudoaneurysm, abscess, ischemia) requiring surgery.

The first patient had undergone mechanical mitral valve replacement. He returned to the clinic 10 days postoperatively with a pulsatile groin mass, and an ultrasound examination revealed a pseudoaneurysm. His anticoagulation medication was withheld, and he returned to the operating room on postoperative day 15 for groin exploration and repair of the femoral artery pseudoaneurysm.

The second patient underwent pulmonary valve replacement and tricuspid valve repair. She returned to the clinic 


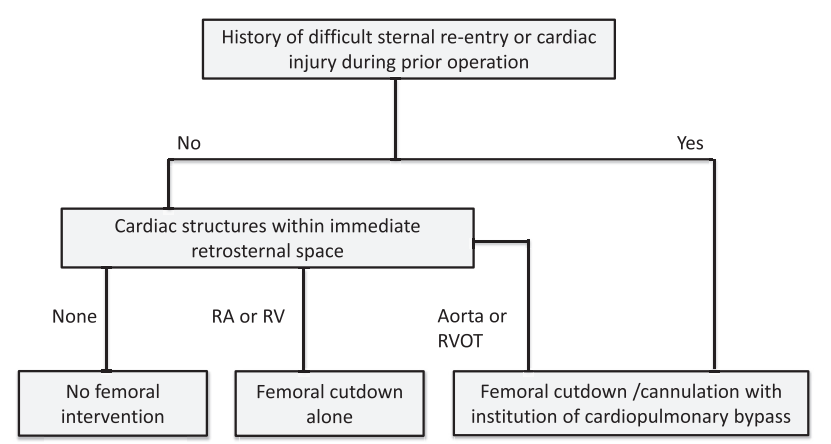

FIGURE 1. Algorithm for presternotomy femoral intervention. $R A$, Right atrium; $R V$, right ventricle; $R V O T$, right ventricular outflow tract.

14 days postoperatively with a groin abscess and underwent surgical drainage and packing in the operating room.

The last patient, who had undergone aortic valve replacement, had a history of smoking and peripheral vascular disease. After extubation on the night of surgery, he complained of left thigh pain. His pulses were weak, and signs of a lower extremity compartment syndrome were present. He returned to the operating room for urgent groin exploration, and a small intimal flap at the femoral cannulation site was repaired.

\section{Outcomes}

With each increase in reoperative sternotomy number, the use of femoral intervention increased (Table 4). Patients who underwent femoral intervention had longer cardiopulmonary bypass times (median, 141 vs 104 minutes; $P=.0002$ ) and longer operative times (median, 388 vs 339 minutes; $P=.005$ ) than those without intervention. Also, the incidence of cardiac injury was greater with the use of femoral intervention $(15 \%$ vs $2 \%, P=.001)$. No differences were found in the incidence of major adverse events or mortality between groups. No patient who sustained a cardiac injury died.

\section{DISCUSSION}

Adults with congenital heart disease are frequently surviving into adulthood, and many of them will require repeat surgery. We have proposed an algorithm using the preoperative history and 3-dimensional imaging findings to decide whether a presternotomy femoral cutdown or cannulation should be performed. A strong correlation was found between the predicted site and the actual site of injury. The risk factors for injury included a history of injury during previous sternotomy, right ventricular outflow tract proximity to the posterior chest wall, and an increased reoperative sternotomy incidence.

\section{Prevention}

Many strategies have been adopted to minimize the risk of sternal reentry, including placement of substernal
TABLE 1. Summary of patient characteristics $(n=205)$

\begin{tabular}{|c|c|}
\hline Variable & Value \\
\hline \multicolumn{2}{|l|}{ Demographics } \\
\hline \multicolumn{2}{|l|}{ Age at surgery $(\mathrm{d})$} \\
\hline Median & 31 \\
\hline Range & $18-76$ \\
\hline \multicolumn{2}{|l|}{ Weight at surgery $(\mathrm{kg})$} \\
\hline Median & 73 \\
\hline Range & $37-141$ \\
\hline \multicolumn{2}{|l|}{ Gender } \\
\hline Male & $105(51)$ \\
\hline Female & $100(49)$ \\
\hline \multicolumn{2}{|l|}{ Reoperative sternotomy (n) } \\
\hline 1 & $113(55)$ \\
\hline 2 & $59(29)$ \\
\hline 3 & $23(11)$ \\
\hline 4 & $10(5)$ \\
\hline \multicolumn{2}{|l|}{ Rationale for presternotomy intervention } \\
\hline History of previous difficult reentry or cardiac injury & $4(2)$ \\
\hline RA proximity & $20(10)$ \\
\hline RV proximity & $49(24)$ \\
\hline RVOT proximity & $35(17)$ \\
\hline Aortic proximity & $4(2)$ \\
\hline \multicolumn{2}{|l|}{ Presternotomy femoral intervention } \\
\hline None & $93(45)$ \\
\hline Femoral cutdown alone & $69(34)$ \\
\hline Femoral cutdown/cannulation with $\mathrm{CPB}$ & $43(21)$ \\
\hline \multicolumn{2}{|l|}{ Operative details } \\
\hline \multicolumn{2}{|l|}{ CPB time (min) } \\
\hline Median & 119 \\
\hline Range & $55-457$ \\
\hline \multicolumn{2}{|l|}{ OR time (min) } \\
\hline Median & 354 \\
\hline Range & $211-968$ \\
\hline \multicolumn{2}{|l|}{ Outcomes } \\
\hline Cardiac injury & $19(9)$ \\
\hline Major adverse event & $41(20)$ \\
\hline Mortality & $5(2)$ \\
\hline
\end{tabular}

protective membranes, presternotomy thoracoscopic dissection of the substernal space, and planning using the preoperative imaging findings.

Placement of a substernal membrane made of expanded polytetrafluoroethylene has been discussed in published studies. Jacobs and colleagues ${ }^{1}$ published a study involving 105 repeat sternotomies after placement of such membranes with 1 injury ( $1 \%$ ) during reentry. None of the patients in our study had a substernal membrane in place at resternotomy. Although we have frequently placed these membranes in both children and adults, the experience in adult congenital heart disease is limited, because many of these patients have not, or will not, return for surgery until decades from now. We hope to evaluate the effectiveness of these protective barriers in reducing the risk of sternal reentry in the future. 


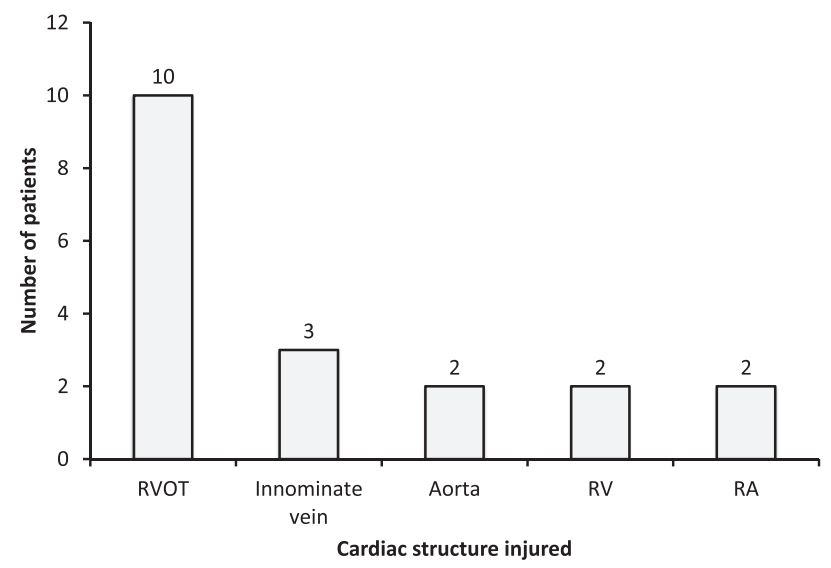

FIGURE 2. Cardiac structure injury pattern. $R A$, Right atrium; $R V$, right ventricle; $R V O T$, right ventricular outflow tract.

Gazzaniga and Palafox ${ }^{2}$ have reported on the use of presternotomy substernal throacoscopic dissection in both pediatric $(\mathrm{n}=12)$ and adult $(\mathrm{n}=39)$ patients. The indications in adult patients were $>1$ previous sternotomy; coronary artery bypass grafting with previous internal mammary artery use; coronary artery bypass grafting with vein grafts immediately behind the sternum; previous aortic, mitral, or tricuspid valve operation; aortic aneurysm; and cardiac enlargement. The indications in pediatric patients were an enlarged heart, previous right ventricular outflow patch or conduit, $\geq 2$ sternotomies, a central shunt, and any previous aortic arch operation.

Aviram and colleagues ${ }^{3}$ evaluated the contribution of multidetector CT angiography in planning repeat cardiac operations. The surgical approach, cannulation site, and myocardial preservation techniques were altered on the basis of the imaging evaluation in several patients. In our study, preoperative 3-dimensional imaging using CT or magnetic resonance imaging was an integral part of the algorithm. In 108 of the 205 patients $(53 \%)$, it led to presternotomy femoral intervention. It was highly predictive of cardiac injury, because 17 of the 19 injuries occurred in this group.
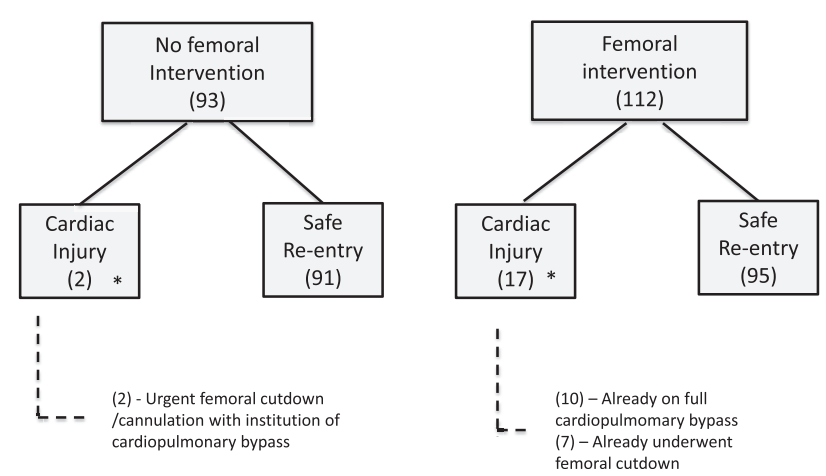

FIGURE 3. Outcome stratified by presternotomy femoral intervention.
TABLE 2. Correlation between actual and predicted sites of injury

\begin{tabular}{llll}
\hline Pt. no. & $\begin{array}{c}\text { Actual site } \\
\text { of injury }\end{array}$ & \multicolumn{1}{c}{$\begin{array}{c}\text { Predicted site of injury } \\
\text { (by preoperative history/ } \\
\text { imaging) }\end{array}$} & Correlation \\
\hline 1 & RA & RA & Yes \\
2 & RA & RA & Yes \\
3 & RV & RV & Yes \\
4 & RV & RV & Yes \\
5 & RVOT & RVOT (by history) & Yes \\
6 & RVOT & RVOT (by history) & Yes \\
7 & RVOT & RVOT (by history) & Yes \\
8 & RVOT & RVOT & Yes \\
9 & RVOT & RVOT & Yes \\
10 & RVOT & RVOT & Yes \\
11 & RVOT & RVOT & Yes \\
12 & RVOT & RVOT & Yes \\
13 & RVOT & RVOT & Yes \\
14 & RVOT & RV & No \\
15 & Aorta & Aorta & Yes \\
16 & Aorta & RV & No \\
17 & Innominate vein & NA & \\
18 & Innominate vein & NA & \\
19 & Innominate vein & NA & \\
\hline
\end{tabular}

Of the 16 patients with information available, the site of injury correlated for 14 $(88 \%)$. Pt. no., Patient number; $R A$, right atrium; $R V$, right ventricle; $R V O T$, right ventricular outflow tract; $N A$, not applicable.

\section{Advantages}

Considering specifically reoperative adult congenital heart surgery, the incidence of cardiac injury in our study was $9 \%$ (19 of 205). Earlier studies of pediatric congenital heart surgery have shown a similar incidence, with a range of $5 \%$ to $10 \%$. ${ }^{4,5}$ However, more recent studies have shown a much lower rate. Morales and colleague ${ }^{6}$ reported a $0.3 \%$ incidence of major injury ( 2 of 602), and Kirshbom and colleagues $^{7}$ reported a $1.3 \%$ incidence in 1000 repeat sternotomies. The greater rate in adults might result from the relative inflexibility of the adult chest wall compared with that in children, making the sternal elevation and substernal exposure and dissection more challenging.

The increased likelihood of cardiac injury with femoral intervention ( $15 \%$ vs $2 \%)$ was likely related to a selection bias. Although the risk of injury was greater, no catastrophic complications developed in those instances in which cardiac injury did occur. Of the 19 patients who sustained a cardiac injury, femoral cutdown had already been performed in 17 patients, of whom 10 had also undergone cannulation. Only 2 patients required urgent femoral cutdown and/or cannulation. Both of these injuries involved the innominate vein. The proximity of the innominate vein to the posterior sternum was not addressed in the preoperative algorithm and did not play a role in the decision to proceed or not with presternotomy femoral intervention. No association was found between the occurrence of a cardiac injury and major adverse event or mortality. It is 
TABLE 3. Univariate risk factor model for cardiac injury

\begin{tabular}{lccc}
\hline Parameter & $\begin{array}{c}\text { No cardiac } \\
\text { injury } \\
(\mathbf{n}=\mathbf{1 8 8})\end{array}$ & $\begin{array}{c}\text { Cardiac } \\
\text { injury } \\
(\mathbf{n}=\mathbf{1 7})\end{array}$ & $\boldsymbol{P}$ value \\
\hline $\begin{array}{l}\text { History of injury } \\
\text { No }\end{array}$ & $187(93)$ & $14(7)$ & $.0001^{*}$ \\
$\quad$ Yes & $1(25)$ & $3(75)$ & \\
RA & & & .89 \\
$\quad$ No & $169(91)$ & $16(9)$ & \\
Yes & $19(95)$ & $1(5)$ & \\
RV & & & .87 \\
No & $141(92)$ & $13(8)$ & \\
Yes & $47(92)$ & $4(8)$ & $.04 *$ \\
RVOT & & & \\
No & $162(94)$ & $11(6)$ & \\
Yes & $26(81)$ & $6(19)$ & \\
Aorta & & & \\
No & $184(92)$ & $16(8)$ & \\
Yes & $4(80)$ & $1(20)$ & \\
Reoperative sternotomy & & & \\
$\quad$ incidence & & & \\
1 & $109(96)$ & $4(4)$ & \\
2 & $53(90)$ & $6(10)$ & \\
3 & $18(78)$ & $5(22)$ & \\
4 & $8(80)$ & $2(20)$ & \\
\hline
\end{tabular}

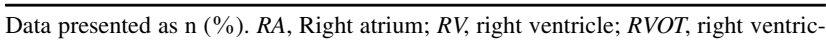
ular outflow tract. *Statistically significant.

unclear whether increased morbidity and mortality would have resulted if the femoral intervention had not been performed.

The other advantage of our liberal use of presternotomy femoral intervention is the flexibility it provides for subsequent cannulation strategies. In patients not already cannulated, the femoral vessels were used as a preferential site of cannulation in 31 additional patients despite safe sternal reentry. The vein $(n=6)$, artery $(n=9)$, or vein and artery $(n=16)$ were cannulated in these patients because of operation-specific anatomic constraints within the chest cavity. Cannulation facilitated aortic surgery $(\mathrm{n}=11)$, right-sided heart dissection in Ebstein's anomaly $(n=2)$, tetralogy of Fallot $(n=9)$, inferior vena cava reconstruction in Fontan revision and/or conversion $(n=7)$, and orthotopic heart transplantation $(n=2)$.

\section{Disadvantages}

The local complications from femoral cannulation included vascular injury (venous or arterial), pseudoaneurysm formation, limb ischemia, groin infection, and groin hematoma. ${ }^{8-10}$ Three of our patients $(1 \%)$ developed groin complications requiring reoperation. All complications were treated successfully, with no permanent damage. Regarding limb ischemia, some investigators have suggested placement of an antegrade superficial femoral artery or a retrograde distal artery
TABLE 4. Comparisons stratified by presternotomy intervention

\begin{tabular}{|c|c|c|c|}
\hline Variable & $\begin{array}{c}\text { No femoral } \\
\text { intervention } \\
(\mathbf{n}=\mathbf{9 3})\end{array}$ & $\begin{array}{c}\text { Femoral cutdown } \\
\text { alone or femoral } \\
\text { cutdown, cannulation, } \\
\text { and } \mathrm{CPB}(\mathrm{n}=112)\end{array}$ & $\begin{array}{c}P \\
\text { value }\end{array}$ \\
\hline \multicolumn{4}{|l|}{ Demographics } \\
\hline Age at surgery (d) & & & .06 \\
\hline Median & 34 & 28 & \\
\hline Range & $18-72$ & $20-76$ & \\
\hline Weight at surgery $(\mathrm{kg})$ & & & .47 \\
\hline Median & 72 & 73 & \\
\hline Range & $40-141$ & $37-122$ & \\
\hline Reoperative sternotomy & & & $.0002 *$ \\
\hline 1 & $65(70)$ & $48(42)$ & \\
\hline 2 & $21(23)$ & $38(34)$ & \\
\hline 3 & $7(8)$ & $16(14)$ & \\
\hline 4 & $0(0)$ & $10(9)$ & \\
\hline \multicolumn{4}{|l|}{ Operative details } \\
\hline CPB time (min) & & & $.0002 *$ \\
\hline Median & 104 & 141 & \\
\hline Range & $59-457$ & $55-403$ & \\
\hline \multicolumn{4}{|l|}{ OR time (min) } \\
\hline Median & 339 & 388 & $.005^{*}$ \\
\hline Range & $211-968$ & $238-738$ & \\
\hline Outcomes & & & $.001^{*}$ \\
\hline Cardiac injury & $2(2)$ & $17(15)$ & \\
\hline Major adverse event & $16(17)$ & $25(22)$ & .39 \\
\hline Mortality & $2(2)$ & $3(2)$ & 1 \\
\hline
\end{tabular}

perfusion catheter to minimize the risk. ${ }^{9,10}$ Beginning in 2009, we began suturing an 8-mm Dacron graft end-to-side onto the common femoral artery during any femoral intervention. Subsequent cannulation of the side graft allowed for bidirectional arterial flow. Our single incident of limb ischemia occurred with direct vessel cannulation before we had adopted this strategy. Systemic complications can also occur from femoral intervention, in particular, events related to air embolism. With prophylactic femoral-femoral bypass, the potential for air embolism exists in patients with an intracardiac shunt who sustain a cardiac injury during sternal reentry or dissection.

In addition to the few complications, femoral intervention also resulted in an increase in the cardiopulmonary bypass time (141 vs 104 minutes, $P=.0002)$ and operative time (388 vs 339 minutes, $P=.005$ ). It is unclear whether these increases were related to the time required for the femoral cutdown and femoral dissection or were a reflection of the complexity of those operations that necessitated presternotomy femoral intervention. However, no increase was found in the incidence of major adverse events or mortality in those patients who had undergone presternotomy femoral intervention compared with those who had not. 
Finally, it is unclear whether additional preoperative imaging should be undertaken to investigate femoral arterial and venous patency. We have chosen not to pursue additional testing. The choice of femoral cutdown side was determined using a combination of previous cardiac catheterization data documenting the patency and size of the vessels and physical examination findings (ie, presence of a previous scar, strength of a palpable pulse, venous varicosities). A right femoral cutdown was performed initially in 46 patients and a left femoral cutdown in 25. A single patient had an unsuitable left femoral artery, and a contralateral cutdown was performed. The axillary artery can also provide an additional site for arterial inflow should neither femoral artery be suitable for cannulation.

\section{Current State}

Overall, cardiac injury during reoperative surgery in adults with congenital heart disease is not uncommon. The preoperative history and preoperative 3-dimensional imaging findings play an important role in the surgical planning of adult congenital reoperation. Most often, the CT scan or magnetic resonance imaging scan already obtained to evaluate the intracardiac pathologic features will suffice to delineate the substernal space. If not, a simple noncontrastenhanced CT scan can be obtained. The findings viewed on these images will predict cardiac injury during sternal reentry and are associated with specific injury patterns.

Because of the universal application of preoperative imaging and the high incidence of subsequent femoral intervention, our approach might be viewed as overly aggressive. A more selective algorithm for presternotomy femoral intervention might be warranted, potentially only performing presternotomy intervention in the 3 high-risk groups-a history of injury during a previous sternotomy, right ventricular outflow tract proximity to the posterior chest wall, and increased reoperative sternotomy incidence.
However, we have chosen to maintain the present preoperative imaging and presterntomy intervention algorithm, despite the low incidence of cardiac injury and despite the lack of an association between cardiac injury and adverse outcomes. Potentially, we were merely fortunate that no adverse outcome ensued in the 2 patients who experienced a cardiac injury who had not undergone presternotomy intervention. It is possible that increased morbidity and mortality would have resulted if interventions had not been performed in those patients who had undergone presternotomy intervention. Finally, we have enjoyed the flexibility that it provides for subsequent cannulation strategies.

\section{References}

1. Jacobs J, Iyer R, Weston J, Amato JJ, Elliot MJ, de Leval MR, et al. Expanded PTFE membrane to prevent cardiac injury during resternotomy for congenital heart disease. Ann Thorac Surg. 1996;62:1778-82.

2. Gazzaniga A, Palafox B. Substernal thoracoscopic guidance during sternal reentry. Ann Thorac Surg. 2001;72:289-90.

3. Aviram G, Sharony R, Kramer A, Nesher N, Loberman D, Ben-Gal Y, et al. Modification of surgical planning based on cardiac multidetector computed tomography in reoperative heart surgery. Ann Thorac Surg. 2005;79:589-95.

4. Russell JL, LeBlanc JG, Sett SS, Potts JE. Risks of repeat sternotomy in pediatric cardiac operations. Ann Thorac Surg. 1998;66:1575-8.

5. Elahi MM, Kirke R, Lee D, Dhannapuneni RR, Hickey MS. The complications of repeat median sternotomy in paediatrics: six-months follow-up of consecutive cases. Interact Cardiovasc Thorac Surg. 2005;4:356-9.

6. Morales D, Zafar F, Arrington K, Gonzalez SM, Mckenzie ED, Heinle JS, et al. Repeat sternotomy in congenital heart surgery: no longer a risk factor. Ann Thorac Surg. 2008;86:897-902.

7. Kirshbom P, Myung R, Simsic J, Kramer Z, Leong T, Kogon B, et al. One thousand repeat sternotomies for congenital cardiac surgery: risk factors for reentry injury. Ann Thorac Surg. 2009;88:158-61.

8. Merin O, Silberman S, Brauner R, Munk Y, Shapira N, Falkowski G, et al. Femoro-femoral bypass for repeat open-heart surgery. Perfusion. 1998;13: 455-9.

9. Gander JW, Fisher JC, Reichstein AR, Gross ER, Aspelund G, Middlesworth W, et al. Limb ischemia after common femoral artery cannulation for venoarterial extracorporeal membrane oxygenation: an unresolved problem. J Pediatr Surg. 2010;45:2136-40.

10. Bisdas T, Beutel G, Warnecke G, Hoeper MM, Kuehn C, Haverich A, et al. Vascular complications in patients undergoing femoral cannulation for extracorporeal membrane oxygen support. Ann Thorac Surg. 2011;92:626-31. 